\title{
Fydlyty: A Low-Fidelity Serious Game for Medical-Based Cultural Competence Education
}

\author{
Zain Khan, Kristopher Maddeaux and Bill Kapralos \\ Faculty of Business and Information Technology \\ University of Ontario Institute of Technology Oshawa, Ontario, Canada. L1H 7K4 \\ Email: zain.khan@uoit.caｋristopher.maddeaux@uoit.net bill.kapralos@uoit.ca
}

\begin{abstract}
Here we present Fydlyty, a web-based, low-fidelity serious game to educate, and inform medical practitioners and trainees about cultural competence. Fydlyty includes a dialogue editor which has the ability to build a conversation, interpret responses, and respond to questions/answers from the game player. These responses are based on predefined cultural characteristics of the virtual character (avatar), and on different moods that the avatar may express depending on the situation (i.e., normal, upset, or angry). In addition to its educational purposes, Fydlyty has been developed as a research tool to examine the role of graphical-based fidelity in the learning process.
\end{abstract}

Keywords-Serious gaming, cultural competence, virtual patient, authoring environment, levels of realism, fidelity.

\section{INTRODUCTION}

The past two decades have witnessed a growing interest in the use of simulation in different industries. Simulation, however is a technique and not a technology [1], having a sole purpose of replicating real-life scenarios in a controlled environment. Replicating real-world scenarios and having learners interact, manipulate, and experiment allows participants to learn, enhance, and sharpen their new or existing skills. Simulation is nothing new and has been used for thousands of years by the military, and health-care practitioners, amongst others. One may criticize the simplicity of the earlier existing simulators or even argue about the fidelity (defined here as the extent to which the appearance and/or the behavior of the simulation matches the appearance and behavior of the real system [2]), but by no means can the facts be neglected that these simulators provided a habitat for the participants in which they acquired new skills or refined their existing ones [3].

Simulations are closely associated with play, a behavior emitted by an individual not motivated by the final result of the behavior [4]. When we formalize play with concrete rules, objectives that push the player to compete, and present a problem that must be solved by taking some action, we call it a game. Games have proven to be a vital educational function for nurturing our learning capabilities and providing us with a solid platform to prepare for real-world scenarios. Moreover, games can also be classified by the components required to play them. One of the branches of games which electronically manipulate images produced by a computer program on a display is known as video games. A subset of videos games known as serious games, have been re-appropriated for educational and training purposes as well [5]. A serious game can formally be defined as an interactive computer application which may or may not have a significant hardware component and comprises of: (i) a challenging goal, (ii) an interface that entertains and/or engages the player, (iii) incorporates concepts of scoring, and (iv) facilitates learning of a new skill or knowledge that can be applied in the real world [6]. In the broadest sense, the fundamental purpose of serious games is to educate the end user [7]. Serious games have been applied to military training [8], health professions education [9], scientific education [10], and business training [11], amongst other areas. Within each of these domains, serious games can be used to facilitate education and training on a wide variety of topics including cultural competence in health-based education [12].

Cultural competence skills include knowing about others cultural values, traditional illnesses, handling language barriers in an adequate manner, and forming a relationship of trust with the patient [13]. Cultural competence, in multicultural societies such as Canada and the United States, is an important skill for health-care practitioners. Moreover, these skills can lead to better communication between the health-care professional and patient, which is very important to avoid patient dissatisfaction, misdiagnosis, and poor outcomes [14]. Despite the importance of cultural competence, it is often missing from medical students' clinical rotations, where it might be most relevant and memorable [15].

Virtual patients (VPs) are defined as interactive computer programs which simulate a real-world scenario between a health-care professional and a patient. During these simulations, learners take on the role of a health-care professional to obtain history, perform a physical exam, and make diagnostic and therapeutic decisions [16]. Even though these simulations are immensely valuable and possess numerous educational benefits, they are limited by their cost and their monotonous nature. As a result, educational and medical institutions often have difficulty creating, maintaining and using these tools [16].

Here we present Fydlyty, a low-fidelity serious game for cultural competence education that includes a novel dialogue authoring system that can be used to develop a wide range of culture-based scenarios. By leveraging the benefits of serious games (i.e., specific challenges or goals, the opportunity to provide instant feedback, and the ability to engage the learner within the learning process), we aim to overcome the limitations of the current approaches and enhance health-based cultural competence education. More specifically, through the use of low fidelity visuals, we ensure the game is easy to develop, and being web-based, it allows the game to run a wide variety of platforms and with minimal computational resources. Furthermore, by employing low-fidelity visuals, it 
allows us to (in the future through comparisons to higher fidelity versions of the game), examine the effect of visual fidelity on learning.

The remainder of the paper is organized as follows. Section 2 provides a brief description of relevant previous work while greater details regarding the Fydlyty serious game are provided in Section 3. Concluding remarks and plans for future work are provided in Section 4.

\section{BACKGROUND}

VPs have been used to improve intercommunication between health-care practitioners and patients. Effective communication between the two, benefits to health-care outcomes, whereas ineffective communication leads to malpractice litigation or even be life threatening for the latter. Stevens et al. [17] developed a system that imitates a standardized patient (SP) to answer questions from the game player who is taking on the role of a medical professional in a particular scenario. The game aims to teach medical students history taking and communication skills in a safe and controllable environment. Similarly, a VP Sita (a 24 year old Indian girl) was created by Sakpal and Wilson [18] to provide students with a better understanding about culture and cultural competence. The goal of the player is to draw answers to a list of already prescribed questions. Both games use a static script-based approach when communicating with the VP. These scripts are only questions hence lack higher order communication skills such as empathy, negotiation, and conveying bad news. Furthermore, there is no framework presented with these approaches, making it is hard to re-use these systems in other contexts of health-care education.

Having limited dialogue scripts is a huge drawback when the intent is to create an immersive virtual environment. To solve this problem, Imison and Hughes [19] proposed a method to enhance the VP's dialogue script by creating low-fidelity, student generated VPs. This method employs existing software to build new VPs. It is cost efficient and can be applied to any form of case-based learning. Even though the approach is very elegant and easy to use, it still has a strong learning curve, it is very long, and involves multiple people who may not always be available for the completion of the process.

\section{The Fydlyty Serious GAME}

Fydlyty is a low-fidelity, cultural competence web-based serious game intended to serve as a tool to teach medical students, practitioners, and professionals cultural competence skills. The goal for the user/trainee is to successfully complete a dialogue script associated with a VP, focusing directly on the mood and the cultural background of the VP. The artificial intelligence (AI) provides a set of predefined responses to the user/trainee, who has to select the most suitable one in reference to the current scenario. Doing so, the system invokes another VP response. Selecting each response correctly allows the user to accumulate further points and move ahead in the conversation until the scenario ends. The system also includes a mechanism to add a set of dialogues to the current database, and to provide the user/trainee feedback based on his/her selections at the end of each scenario.

\section{A. Overview}

For the sake of simplicity and better understanding, we have chosen to illustrate the game with a specific scenario whereby the player, taking on the role of the health-care provider (a physician in this particular scenario), begins the game to examine an elderly female VP, Li Chen, sitting in the clinic. The virtual world is viewed through the user's point-of-view (e.g., first person perspective) and no part of the player's body is visible during the course of the game. A conventional mouse is the primary source of input from the user to the system. The screengrab of the index page shown in Fig. 1, depicts the VP on the left of the users screen and on the right is a short introduction of the game, motivation behind it, objectives, and cues of how to proceed forward in the game. This basic information helps the user familiarize him/herself with the game environment before starting the actual game.

After the introduction and explanation of the game rules, the game proceeds to the next page (see Fig. 2). This is the first phase of the game where the system starts with providing the player with the preliminary description of the patient. These traits include: (i) name of the patient, (ii) location at where the patient is being treated, (iii) informant who provided the privileged information regarding the patient, (iv) chief complaint that the patient endures, and (v) history of present illness.

The patient information provided to the player before the conversation begins, is based on the standard history and examination guide used in medical schools [20]. We have selected a sub-set of this information (five factors) that is relevant to cultural competence education: (i) name, (ii) location, (iii) informant, (iv) chief complaint, and (v) history of present illness, which will help the player acquire the patient's personal and medical information. The patient information page initially shows the name of the patient, so that while conversing the player can connect with his/her subject. Players in the game can make mistakes by referring to the patient with the incorrect name thus potentially offending the patient. The location is another attribute which may help the player be more aware about his/her surroundings. The informant tells the player of the person who provided information regarding the patient's health; this can be anyone among (e.g., family, and/or friends with the patient, or the patient him/herself). Further, the chief complaint field indicates the purpose of the visit and the primary problem that the patient suffers from. In the example shown, this is the third visit for this 83 year old woman with a long history of hypertension. The last section provides the history of the present illness and provides information regarding the specific patient complaint.

After obtaining the patient information, the player proceeds by clicking on the 'Start Conversing' button at the bottom of the page. From this point onwards, the game is timed and all choices made by the user/trainee add to the success/failure of the conversation. Fig. 3 shows the different components comprising the game page. At the top of the page the time elapsed is recorded (labeled at A in Fig. 3). The timer provides one form of feedback at the end of the game and also initiates a sense of urgency in the player. Below the clock, is the patient console (labeled at B in Fig. 3). The patient console shows automated replies from the VP which are illustrated for the trainee to read and accordingly choose his/her next reply 


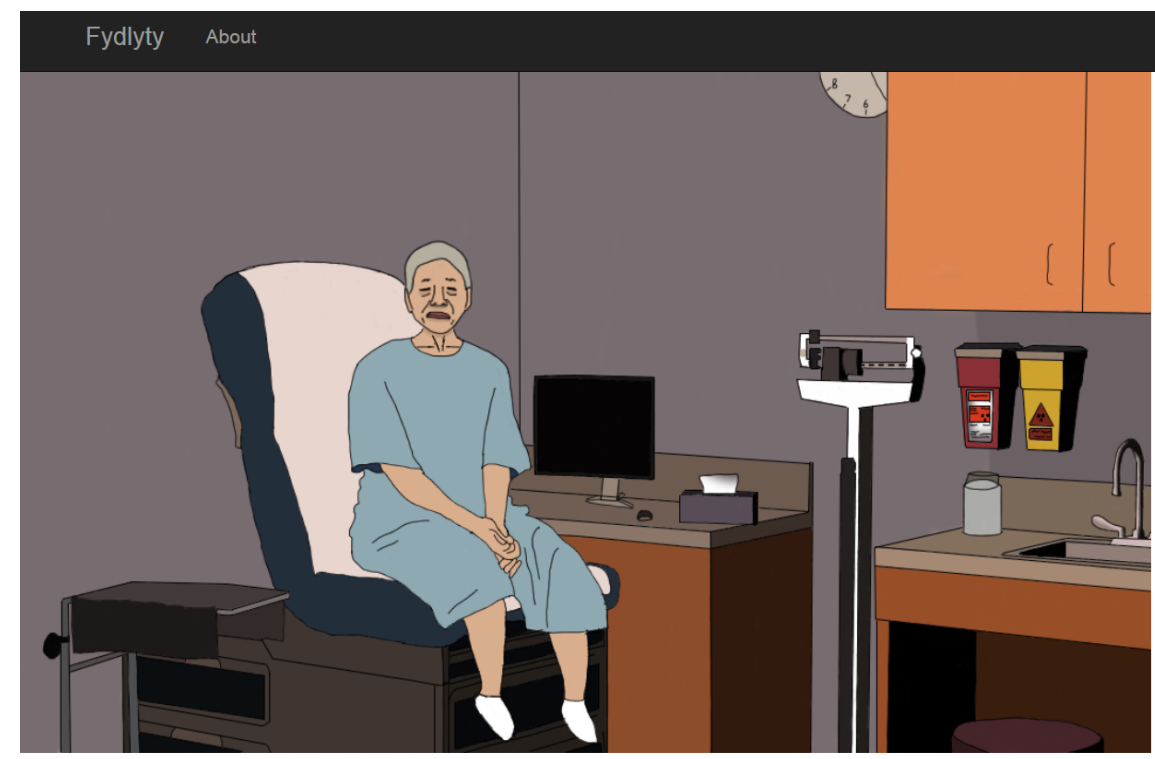

\section{Welcome to Fydlyty}

A Low-fidelity Cultural Competence Serious Game

Fydlyty is a low-fidelity cultural competence web-based game. The dem shows a virtual patient, Li Chen sitting in the clinic. The game starts with the description of the patient provided to the game player. These traits include the age of the patient, gender, ethnicity, etc. After this the player will be given a bunch of options out of which he/she would have to pick one. These options will be questions, actions or responses for the VP. The VP will have a counter response to the option selected. This response can be verbal (e.g., an answer to the question) or physical (e.g., change in the mood). The dialogue script can be used to create new scenarios. As the conversation proceeds, the VP will develop an opinion about the player which is done by using our impression formation algorithm. This opinion along with the score will be shown to the user at the end of each game.

How to play the game

- Fydlyty is a conversation between a doctor and a patient

- When you start the game as a doctor to Li, some prior information is given about her medical conditions

- After starting you will be given multiple dialogue scripts, out of which you have to click on the best reply to $L I$

\section{Check Patient Information}

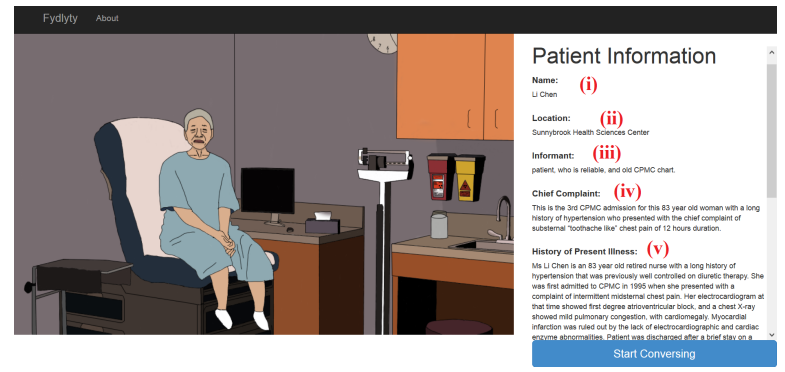

Fig. 2: Patient Information page shows information regarding patient $\mathrm{Li}$ Chen's medical problem.

to. The next section (labeled at C in Fig. 3), lists a pool of potential replies for the VP. The user can choose one of the many replies by simply clicking on to the desired choice. These options provided can be questions, actions, or responses for the VP. The VP will have a counter response to the selected option. This response can be verbal (e.g., an answer to the question) or physical (e.g., change in the mood). Verbal responses will be shown in the patient console, whereas, physical responses will be depicted by the change in facial expressions on the VPs illustration.

When the game begins, the system randomly selects either the VP or the game player to start the conversation. Both of these characters have a pool of dialogues which they can use to begin the conversation. For example, the doctor when initiating the conversation with the patient may choose to say "Hello. I am Dr. Smith. I will be the physician taking care of you today. What seems to be the problem?". If the VP initiates the conversation, it may begin with "Hello doctor! Are you the physician for today's session?". In reference to the system's terminology, these dialogues are called 'initiators'. An initiator is a dialogue which is used to begin a conversation and will be the root of the script, thus having no prior dialogues associated

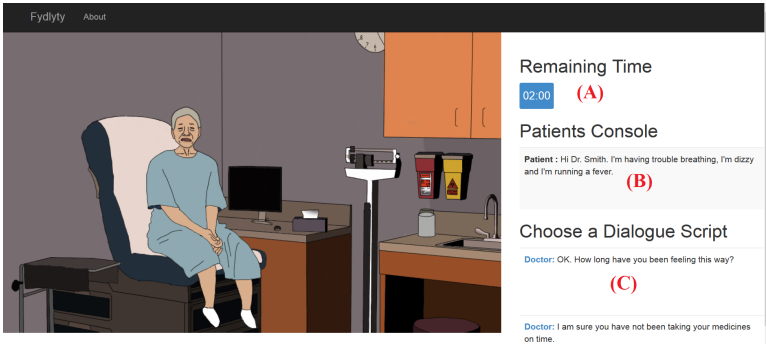

Fig. 3: Timer (A), Patient Console (B), and Choices for Dialogue Script (C) comprise of the game page.

to it. This is described in greater detail below.

In the event that the VP is selected to initiate the conversation, the system will return a randomly selected dialogue associated with the patient and a couple of options related to the doctor. These options are related to the patient's initial dialogue and will build the conversation forward. Selecting an option from the list will invoke another doctor-patient dialogue set. These new dialogues are now from the next level of the dialogue hierarchy. As the player proceeds in the game, the number of options available to them increases. Here the system exhibits the concept of fading, where the task steadily starts to become more difficult for the user, testing his/her response time and their command over the particular skill set.

Each sentence stored in the database has a particular type of mood associated with it. The VP exhibits three basic moods: (i) normal, (ii) upset, and (iii) angry. The choice of sentence selected by the player will vary the mood of the VP. For example, if the VP says "I'm having trouble breathing, I'm dizzy and I'm running a fever." to what the player responds by saying "I am sure you have not been taking your medicines on time.", there is a high probability that the patient will not ap- 
preciate this reaction by the doctor (player in our scenario) and consider this statement as an accusation, therefore, upsetting the VP. The goal of the game is to successfully complete the scenario without upsetting the VP and maintain a relationship so that the VP may visit the same physician again. If during the conversation things don't go well and the mood of the VP becomes angry, the VP requests to see another physician. At this point the game ends and the unsuccessful user is sent to the feedback page.

The feedback page is shown in Fig. 4, where the transcript of the conversation between the trainee and VP is listed. Each option selected by the trainee has a particular mood related to it i.e. normal, upset, or angry, represented by yellow, green, and red respectively, on the feedback page. By going through the transcript, the player can observe the affect of the responses on the conversation, and the mistakes he/she made. The feedback page also shows the time taken by the user to complete the scenario. As shows in Fig. 4, we can see that the player completed the scenario in four minutes and that the initial conversation between the VP and the user went very well (highlighted as green), but as soon as the user burdened the VP by telling her about her illness and the seriousness of it, this upset her (highlighted as yellow). The game player recovered again by showing concern towards the VP but aggravated the situation when talking about financial matters (highlighted as red).

Fydylyty supports two types of dialogue: (i) doctor or (ii) patient. Both types are saved in the database and follow the same structure. As shown in Fig. 5, an instance of the dialogue will have a recursive relationship with itself and will comprise of four attributes: (i) parent, (ii) utterance, (iii) character, and (iv) mood. The parent attribute is a foreign key relationship with another dialogue entity. This relationship forms the structure of the conversation. Each dialogue will have one or no parent. A null parent signifies that the entity can be selected to initiate the conversation by either the patient or the doctor. Utterance is the actual text of the dialogue which is rendered during the game. Each dialogue represents either patient or the doctor in the game, therefore, the character attribute stores the type of character with whom the dialogue is associated with. Finally, the mood attribute saves the affect of choosing the dialogue on the patient. The mood can be normal, upset, or angry. This field can also be empty, indicating no repercussions during the game after choosing that instance.

\section{B. Technical Details}

Fydlyty was developed using Django. Django is a high-level web framework built using the Python programming language. The framework encourages rapid-application development with a clean and pragmatic design. The illustrations shown in the game were created "in-house" and the rendering at the frontend is done using Twitter Bootstrap. Bootstrap is a popular front-end frameworks for developing responsive designs. Bootstrap provides Fydlyty the power to run on different screen resolutions and browsers without having to worry about cross-browser compatibility, inconsistent interface design, and unfriendly screen resolution. The entire game is intended to be played in a single session, thus Ajax is an integral part of the system. Ajax is used on the client-side to create asynchronous web applications and is attained using jQuery. To store the

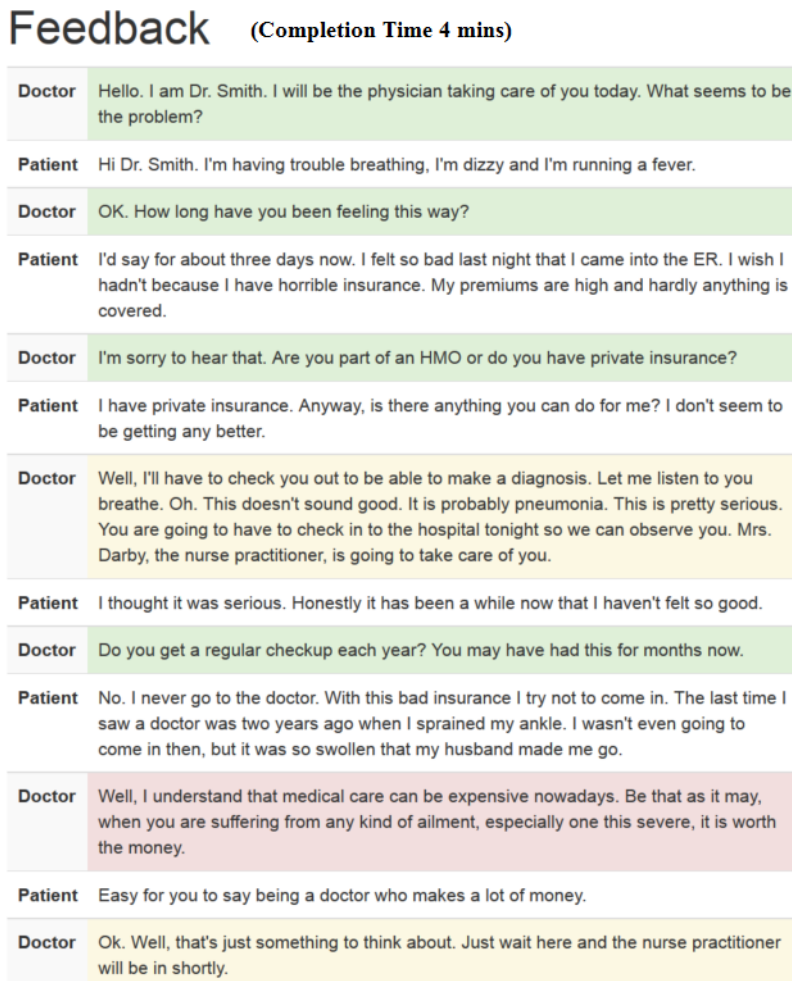

Fig. 4: Feedback page shows the transcript of the conversation between the VP and the user.

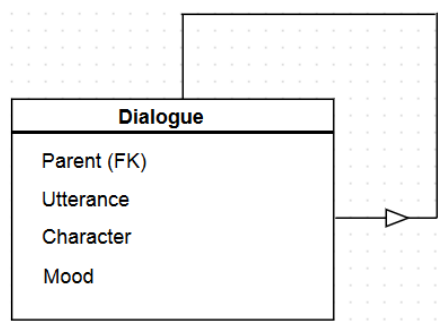

Fig. 5: A recursive relationship of the dialogue entity builds a conversation tree.

dialogues in the system, a relational database management system, SQLite is also used.

\section{CONCLusion And Future Work}

Designers and developers of serious games and virtual simulations in general, typically strive for high-fidelity environments, particularly with respect to the visual (graphical) scene. However, despite the great computing hardware and computational advances we have experienced, real-time high fidelity rendering of complex environments (found in many serious games) across all modalities is still not feasible [21]. Furthermore, recent evidence suggests high fidelity simulation does not always lead to greater learning [22], and striving for high-fidelity can burden our computational resources (particularly when considering portable computing devices), and lead to increased development costs. Although when considering multi-modal virtual learning environments, many factors can 
influence graphical fidelity perception (e.g., sound), for the purpose of this work, we are only considering the visual scene. The results of this work will form the basis of further, more extensive experiments that will examine the role of multimodal interactions on visual fidelity perception.

Here we presented Fydlyty, a low visual fidelity, web-based serious game for medical-based cultural competence education. Fydlyty allows for a wide variety of scenarios to be easily developed and/or edited using a simple scenario editor, ensuring it is practical across a wide variety of areas and applications. We also outlined a specific scenario where a VP, Li Chen, had a medical ailment and thus visited her doctor. At this point the conversation between the VP and the player (taking on the role of the doctor) begins. Depending on the player's response, during the conversation the mood of the VP may change (e.g., become upset, angry, etc.). The player's goal in the game is to ensure they maintain a good relation with the patient, so that the patient will visit him/her again. At the end of each session, feedback is also provided so that the trainee is informed about the mistakes they made and how they could be corrected. Although formal user-based evaluations have not been conducted yet, an informal test session with three Computer Science graduate students at the University of Ontario Institute of Technology indicate that Fydlyty is well designed, intuitive, and that its objectives were easy to understand at the first attempt at playing the game. The informal test session also pointed out that it would be helpful to have multiple patients (scenarios) to reflect a greater variety of cultural-based scenarios and one of the participants suggested that the game requires greater challenge perhaps through a more complex scenario (this is actually not a limitation of Fydlyty; a scenario can be made arbitrarily complex but here a simple one was chosen for illustration purposes).

Future work will examine the integration of Fydlyty into the medical curriculum and include a more thorough user-based study to better gauge the effectiveness of the serious game. Furthermore, the feedback provided at the end of the game will be improved so that the trainee may examine the list of favorable options he/she could have selected. This will help the player to examine different approaches of completing each scenario in a more beneficial way. Future work will also involve using Fydlyty to examine whet, if any effect low fidelity visuals (graphics) have on learning. This will be accomplished through a pre- and post-testing study that will include three groups of participants: (i) control group which will not use Fydlyty but will recieve all of the educational material using traditional methods, (ii) a group that will use the low-fidelity version of Fydlyty, and (iii) a group that will Fydlyty with high-fidelity graphics.

\section{ACKNOWLEDGMENT}

The authors would like to thank Rukham Ajaz at Jinnah Hospital Lahore. This work was supported by an Ontario Simulation Network (SIM-one) Simulation Research \& Innovation Grant. The support of the Natural Sciences and Engineering Research Council of Canada (NSERC), the Social Sciences and Humanities Research Council of Canada (SSHRC), in support of the IMMERSe project, and the Canadian Network of Centres of Excellence (NCE) in support of the GRAND initiative is also acknowledged.

\section{REFERENCES}

[1] D. M. Gaba, "The future vision of simulation in health care," Quality and Safety in Health Care 2004, vol. 13, no. suppl 1, pp. i2-i10.

[2] E. Farmer, J. von Rooij, J. Riemersma, P. Joma, and J. Morall, Handbook of simulator based training. Ashgate Publishing Limited, Surrey, UK, 1999.

[3] W. R. Boot, A. F. Kramer, D. D. J. Simons, M. Fabiani, and G. Gratton, "The effects of video game playing on attention, memory, and executive control," Acta Psychologica 2008, vol. 129, no. 3, pp. 387-398.

[4] M. J. Ellis, "Why people play," 1973.

[5] C. van Est, R. Poelman, and R. Bidarra, "High-level scenario editing for serious games.," in GRAPP, pp. 339-346, 2011.

[6] B. Bergeron, "Developing serious games (Game Development Series)," 2006.

[7] D. G. Frederik, M. Peter, and V. L. Jan, "Uncharted waters?: exploring experts' opinions on the opportunities and limitations of serious games for foreign language learning," in Proceedings of the 3rd International Conference on Fun and Games 2010, pp. 107-115.

[8] W. L. Johnson and A. Valente, "Collaborative authoring of serious games for language and culture," in Proceedings of SimTecT 2008.

[9] Q. Wang, O. Sourina, and M. K. Nguyen, "Eeg-based 'serious' games design for medical applications," in IEEE International Conference on Cyberworlds 2010, pp. 270-276.

[10] K. Millis, C. Forsyth, H. Butler, P. Wallace, A. Graesser, and D. Halpern, "Operation aries!: A serious game for teaching scientific inquiry," in Serious games and edutainment applications 2011, pp. 169195.

[11] T. Lainema and P. Makkonen, "Applying constructivist approach to educational business games: Case realgame," Simulation \& gaming 2003, vol. 34, no. 1, pp. 131-149.

[12] M. Beig, A. Mayer, C. Chan, B. Kapralos, and A. Dubrowski, "A serious game for medical-based cultural competence education and training," in IEEE 14th International Conference on Advanced Learning Technologies (ICALT), 2014, pp. 211-212.

[13] G. Flores, "Culture and the patient-physician relationship: achieving cultural competency in health care," The Journal of pediatrics 2000, vol. 136, no. 1, pp. 14-23.

[14] B. M. Korsch, E. K. Gozzi, and V. Francis, "Gaps in doctor-patient communication i. doctor-patient interaction and patient satisfaction," Pediatrics 1968, vol. 42, no. 5, pp. 855-871.

[15] S. Kripalani, J. Bussey-Jones, M. G. Katz, and I. Genao, "A prescription for cultural competence in medical education," Journal of General Internal Medicine 2006.

[16] G. Huang, R. Reynolds, and C. Candler, "Virtual patient simulation at US and Canadian medical schools," Academic Medicine 2007, vol. 82, no. 5, pp. 446-451.

[17] A. Stevens, J. Hernandez, K. Johnsen, R. Dickerson, A. Raij, C. Harrison, M. DiPietro, B. Allen, R. Ferdig, S. Foti, et al., "The use of virtual patients to teach medical students history taking and communication skills," The American Journal of Surgery 2006, vol. 191, no. 6, pp. 806811.

[18] R. Sakpal and D. Wilson, "Can culture translate to the virtual world?," in HCI International-Posters Extended Abstracts 2011, pp. 242-246.

[19] M. Imison and C. Hughes, "The virtual patient project: Using low fidelity, student generated online cases in medical education," in Australian Society for Computers in Learning in Tertiary Education Annual Conference (ASCILITE), 2008, pp. 441-445.

[20] V. L. Patel, A. W. Kushniruk, S. Yang, and J. Yale, "Impact of a computer-based patient record system on data collection, knowledge organization, and reasoning," Journal of the American Medical Informatics Association 2000, vol. 7, no. 6, pp. 569-585.

[21] V. Hulusic, C. Harvey, K. Debattista, N. Tsingos, S. Walker, D. Howard, and A. Chalmers, "Acoustic rendering and auditory-visual cross-modal perception and interaction," in Computer Graphics Forum 2012, vol. 31, pp. 102-131.

[22] G. Norman, K. Dore, and L. Grierson, "The minimal relationship between simulation fidelity and transfer of learning," Medical education 2012, vol. 46, no. 7, pp. 636-647. 